\title{
Fatiga de contacto por rodadura en acero SAE 1050 recubierto con TiN, posteriormente templado
}

\author{
Rolling contact fatigue of quenched TiN \\ coated SAE 1050 steel
}

Alejo Daniel Mandri ${ }^{1,2}$, Sebastián Laino ${ }^{1,2}$,
Ricardo César Dommarco

\author{
${ }^{1}$ Grupo Tribología - Facultad de Ingeniería - Universidad Nacional de Mar del Plata, Mar del Plata, Buenos Aires, Ar- \\ gentina. \\ ${ }^{2}$ Div. Metalurgia - INTEMA - CONICET CP: 7600, Mar del Plata, Buenos Aires, Argentina. \\ e-mail: alejomandri@fi.mdp.edu.ar; slaino@fi.mdp.edu.ar; dommarco@fi.mdp.edu.ar
}

\section{RESUMEN}

La aplicación de recubrimientos cerámicos del tipo nitruros, carburos o diamante, sobre sustratos de acero aporta diversas bondades tribológicas. Sin embargo elevados gradientes de dureza y problemas de adhesión con el sustrato pueden perjudicar el desempeño de estos recubrimientos frente a la Fatiga de Contacto por Rodadura (FCR). Numerosos estudios realizados sobre aceros que mantienen la dureza a elevadas temperaturas, recubiertos con films cerámicos, se han enfocado en esta cuestión. En el presente trabajo se estudia el uso de un acero de bajo costo, como el SAE 1050, con un tratamiento superficial combinado, consistente en un recubrimiento de TiN por Deposición Física de Vapor (PVD), y un temple superficial posterior por inducción que proporcione al sustrato la resistencia sub-superficial necesaria para soportar las tensiones del macrocontacto. La secuencia de tratamientos propuesta, a diferencia de la usualmente empleada (temple+PVD), pretende mantener la dureza de temple del sustrato, aunque resulten inciertas las consecuencias sobre el recubrimiento. Los ensayos para evaluar la resistencia a la FCR se realizaron con una máquina tipo arandela plana, en la cual una probeta en forma de disco gira sobre un conjunto de bolillas correspondientes a un rodamiento de empuje axial, bajo la acción de una carga normal. Los resultados fueron analizados de manera estadística y el daño resultante fue observado con microscopio óptico y microscopio electrónico de barrido (SEM), en complemento con mapeos de espectrometría de dispersión de energía de rayos X (EDS). En el caso estudiado, el tratamiento realizado permitió obtener algunas de las ventajas típicas de los recubrimientos, sobre todo aquellas relacionadas con la fricción y el desgaste adhesivo y abrasivo, sin una drástica modificación en la vida a la FCR, en sustratos de aceros de construcción. También se realizaron ensayos de Difracción de rayos X (DRX) para evaluar la modificación del recubrimiento como consecuencia del temple superficial.

Palabras clave: Desgaste, FCR, acero, recubrimiento, TiN.

\begin{abstract}
The use of ceramic coatings of the nitride, carbide, or diamond type on steel substrates promotes the improvement of tribology properties. Nevertheless, high hardness gradients and adhesion problems may deteriorate the performance of these coatings when subjected to Rolling Contact Fatigue (RCF). Some studies on steels with ceramic coatings were carried out on steels that sustain hardness at elevated temperature. In the present study, a low cost steel, SAE 1050 was used with a combined surface treatment consisting of a TiN Physical Vapor Deposited (PVD) coating plus a subsequent induction hardening, in order to give the coating high substrate hardness, necessary to withstand the contact stresses. The proposed treatment sequence (different to that usually employed as quenching + PVD), was intended to sustain the high hardness of the quenched steel, even though the consequences on the previous PVD coating are unknown. The tests to evaluate RCF were carried out with a washer type fatigue tester in which a disc sample is rotated under load against a thrust bearing acting as a counterpart. All the system is lubricated by immersion in ISO VG 100 industrial oil. The RCF life values for the individual tests were statistically analyzed and the wear tracks were studied by optical and scanning electron microscopy. Also mappings by X-ray dispersive spectroscopy (EDS) were carried out. The proposed sequence for the surface treatment allowed to obtain some of the typical im-
\end{abstract}


provements for coatings as for example those related to low friction, adhesion and abrasion resistance, without impairing the RCF resistance. These benefits are possible even tough plain medium carbon steel was used. $\mathrm{X}$ ray diffraction analyses were also carried out in order to evaluate the coating degradation after quenching.

Keywords: Wear, RCF, steel, coating, TiN.

\section{INTRODUCCIÓN}

El desgaste es reconocido como uno de los más importantes procesos de deterioro en elementos mecánicos [1]. Si bien el desgaste no es una falla catastrófica, se trata de un proceso de deterioro que reduce gradualmente la eficiencia y, finalmente, conduce a la salida de servicio de la maquinaria. El importante crecimiento de la tribología en las últimas décadas se debe, por un lado, al desarrollo de otras ciencias paralelas, como la de los materiales, lo cual ha permitido un control eficaz de las fallas de tipo catastrófico y, por otro lado, a la necesidad de hacer un uso racional de los recursos naturales y eficiente de los elementos de máquina. Esto ha hecho que hoy en día, una de las principales causas para la salida de servicio de los equipos o componentes de máquina sea el desgaste.

La norma ASTM G 40 [2] define a la fatiga de contacto por rodadura (FCR), como un proceso de daño que tiene lugar en un tribo-elemento sujeto a cargas repetidas, originadas en el contacto por rodadura, que involucra la nucleación de una fisura (en o debajo de la superficie de contacto) y su propagación, siendo un proceso que culmina con la formación de pits o picaduras superficiales, o cráteres, dependiendo de su tamaño.

Numerosos componentes utilizados en automóviles, máquinas y herramientas, dedicados a la transmisión de movimiento y esfuerzos, soportan la aplicación de cargas elevadas y variables en el tiempo, en áreas de contacto reducidas, dando lugar a este mecanismo de desgaste. El ejemplo más común lo constituyen los rodamientos, que usualmente son fabricados en aceros de alta dureza con un tratamiento térmico a corazón. Otros ejemplos de piezas sometidas a FCR son: sistema leva-seguidor, rueda-riel en el ferrocarril, cilindros de laminación, engranajes, etc.

En la actualidad, las crecientes exigencias impuestas a los componentes mecánicos conducen frecuentemente a la ruptura de la película de lubricante que debiera separar las dos superficies de trabajo, estableciéndose el contacto metal - metal. En tal situación, las máximas tensiones originadas en torno a los micro contactos resultan notablemente superiores a aquellas originadas en condiciones de lubricación fluida, y se encuentran además, mucho más próximas a las superficies, derivando en un tipo particular de falla conocida como superficial temprana.

Usualmente este tipo de daño ha sido combatido a través de tratamientos termoquímicos y tratamientos térmicos, que confieran al material la aptitud para soportar los esfuerzos superficiales originados. Puesto que en piezas de gran tamaño se vuelve dificultoso el tratamiento térmico a corazón, suele optarse por el uso de tratamientos superficiales, siendo ejemplos típicos el temple superficial, la cementación y la nitruración gaseosa. Estos dos últimos tratamientos, si bien proveen resultados satisfactorios, implican también un costo de procesamiento elevado.

Por otro lado el avance en el procesamiento superficial de materiales asistido por plasma permite disponer de diversas técnicas de recubrimiento y modificación superficial a costos industriales competitivos. Mediante la técnica de deposición física de vapor (PVD), es posible depositar gran variedad de recubrimientos, desde metales puros o aleaciones, hasta cerámicos tales como nitruros, carburos o carbo - nitruros. En particular, el grupo de los recubrimientos cerámicos es muy utilizado en componentes mecánicos, para proporcionar mejoras tribológicas a la superficie, reduciendo el coeficiente de fricción, el desgaste producido por ciertos mecanismos, mejorar la resistencia a la corrosión, y realzando el aspecto de las piezas a través del brillo y la tonalidad.

La alta dureza obtenible con estos recubrimientos, posibilita pensar en su uso para soportar esfuerzos superficiales originados en situaciones de carga concentrada como la FCR, aprovechando además otras ventajas tribológicas. De cualquier manera, es necesario que el sustrato posea una alta dureza, dado que las máximas tensiones de corte originadas en tales situaciones, tienen lugar en la sub-superficie, por debajo de la capa depositada. De esa forma los recubrimientos son mejor soportados y flexionan menos, reduciendo la cantidad de fisuras que podrían aparecer, conduciendo finalmente a un desprendimiento parcial.

Diversos autores $[3,4,5]$ han estudiado el comportamiento a la FCR de diferentes aceros recubiertos con materiales tales como nitruro de titanio $(\mathrm{TiN})$, nitruro de cromo $(\mathrm{CrN})$, carbonitruro de titanio $(\mathrm{Ti}(\mathrm{CN}))$ y diamond like carbon (DLC). Los estudios se han enfocado principalmente en aceros aleados, que mantienen la dureza a la temperatura y tiempo para los cuales los recubrimientos son depositados, entre 300 y $400{ }^{\circ} \mathrm{C}$ durante algunas horas. 
El presente trabajo contempla el estudio de la resistencia a la FCR de un acero de bajo costo, como el SAE 1050, con un tratamiento superficial combinado, consistente en un recubrimiento de TiN por PVD, que provea una mayor resistencia en la superficie, y un temple superficial posterior por inducción, que proporcione al material la resistencia sub-superficial necesaria para soportar las tensiones del macro-contacto. Cabe destacar que la secuencia de tratamientos propuesta, a diferencia de las usualmente empleadas, pretende mantener la dureza de temple del sustrato, a costa del posible deterioro que el recubrimiento pudiera sufrir durante ese proceso, siendo también motivo de estudio en el presente trabajo. Los resultados de vida hasta la falla obtenidos son analizados en forma estadística, y comparados con los de probetas del mismo acero, pero sometidas sólo al tratamiento de temple por inducción. El daño resultante también fue observado con microscopio óptico y microscopio electrónico de barrido (SEM), en complemento con mapeos de espectrometría de dispersión de energía de rayos X (EDS).

\section{MATERIALES Y MÉTODOS}

Todas las probetas de FCR utilizadas en este trabajo fueron mecanizadas a partir de una misma barra redonda de acero SAE 1050 de $75 \mathrm{~mm}$ de diámetro. Las superficies planas fueron rectificadas con piedra y terminadas manualmente con lija de grano 100, eliminándose las marcas del mecanizado previo. Posteriormente fueron divididas en dos grupos (A y B) que recibieron distintos tratamientos realizados por la empresa Sudosilo S.A. El grupo A fue sometido a temple superficial por inducción, mientras que en el Grupo B se depositó por PVD un recubrimiento de TiN de $1 \mu \mathrm{m}$ de espesor, y luego se realizó el temple superficial por inducción. La deposición del recubrimiento fue realizada durante 1 hora, mediante un proceso de PVD reactivo, asistido por plasma generado por arco, con una temperatura de sustrato de $350{ }^{\circ} \mathrm{C}$. Luego, se realizó el temple superficial por inducción con una frecuencia de $450 \mathrm{kHz}$ y una potencia de $60 \mathrm{~kW}$, empleando un inductor plano en espiral. La temperatura de austenizado fue de entre 880 y $900{ }^{\circ} \mathrm{C}$ con un tiempo de calentamiento de 11 segundos y un posterior enfriamiento bajo una ducha de agua a $25{ }^{\circ} \mathrm{C}$ durante 5 segundos. Luego se realizó un tratamiento de distensionado manteniendo las probetas a $180{ }^{\circ} \mathrm{C}$ durante una hora. Las superficies recubiertas con TiN no requirieron ninguna preparación adicional posterior al tratamiento térmico, en tanto que aquellas superficies sin recubrimiento, que solamente fueron templadas, requirieron un desbaste manual con lija de granulometría 100 para eliminar la oxidación superficial producida durante tal proceso.

La rugosidad media aritmética, Ra, se mantuvo dentro del rango de 0,3-0,6 $\mu \mathrm{m}$ para todas las probetas estudiadas, en tanto que las bolillas poseen un $\mathrm{Ra}=0,015 \mu \mathrm{m}$. La dureza obtenida en la superficie de las muestras resultó de $\sim 56 \mathrm{HRC}$. El espesor de los recubrimientos fue medido por microscopia óptica, previa preparación de la sección transversal de una muestra testigo y también por el método Calotest. De acuerdo con lo calculado a través de las expresiones desarrolladas por Hamrock y Dowson [6] el espesor de película lubricante, $\mathrm{h}_{0}$, resultó de $0,56 \mu \mathrm{m}$ y $0,50 \mu \mathrm{m}$ para los ensayos realizados con rodamientos de empuje axial de $44 \mathrm{~mm}$ y $38,5 \mathrm{~mm}$ de diámetro, respectivamente. En tanto que el coeficiente de espesor de película lubricante o factor Lambda $(\lambda)$, calculado de acuerdo a la ecuación 1, resultó de 0,94 y 1,05, respectivamente, valor que representa que la condición de ensayo promueve el contacto entre micro asperezas y, por lo tanto, la falla superficial temprana. Los valores de $\sigma_{1}$ y $\sigma_{2}$ corresponden a la rugosidad media aritmética de los dos cuerpos en contacto (probeta y bolilla).

$$
\lambda=\frac{h_{0}}{\sigma}=\frac{h_{0}}{\sqrt{\sigma_{1}^{2}+\sigma_{2}^{2}}}
$$

Los ensayos para evaluar la resistencia a la FCR se realizaron con una máquina tipo arandela plana, en la cual una probeta en forma de disco gira sobre un conjunto de bolillas correspondientes a un rodamiento de empuje axial, bajo la acción de una carga normal. En tales condiciones, cada punto de la probeta perteneciente al camino de rodadura (CR), se encuentra sometido al paso repetido de los elementos rodantes, y por lo tanto a la acción variable de una carga. La Fig. 1 esquematiza la configuración utilizada. El conjunto trabaja sumergido dentro de una cuba de aceite hidráulico ISO VG 100, bajo un régimen de lubricación mixto que trae aparejado el contacto entre micro asperezas promoviendo la falla superficial temprana. Los ensayos fueron finalizados al desarrollarse un cráter de igual dimensión que el ancho del $\mathrm{CR}$, registrándose la cantidad de ciclos de carga transcurridos hasta entonces.

La carga aplicada sobre la probeta fue de $304 \mathrm{~N}$ y estuvo soportada por cinco bolillas de rodamiento de $6 \mathrm{~mm}$ de diámetro, dispuestas sobre contra muestras de 38,5 mm y $44 \mathrm{~mm}$ de diámetro, rodamientos tipo 51106 y 51107, respectivamente. En cada punto o área de contacto se origina una distribución de presiones (normal a la superficie) con un valor máximo de $\mathrm{p}_{0}=2,6 \mathrm{GPa}$, de acuerdo con la teoría de contacto lineal elástica de Hertz. La velocidad de rotación fue de 1675 r.p.m., en esta situación cada punto del CR experi- 
menta una cantidad de $2,51 \times 10^{5}$ ciclos de carga por cada hora de ensayo. Para detectar el momento de la falla, la máquina cuenta con un sensor de vibraciones, que detiene el ensayo al superarse cierto umbral permitido. Adicionalmente, las probetas fueron examinadas de forma periódica durante los ensayos para observar el estado del CR y la integridad de los elementos rodantes.

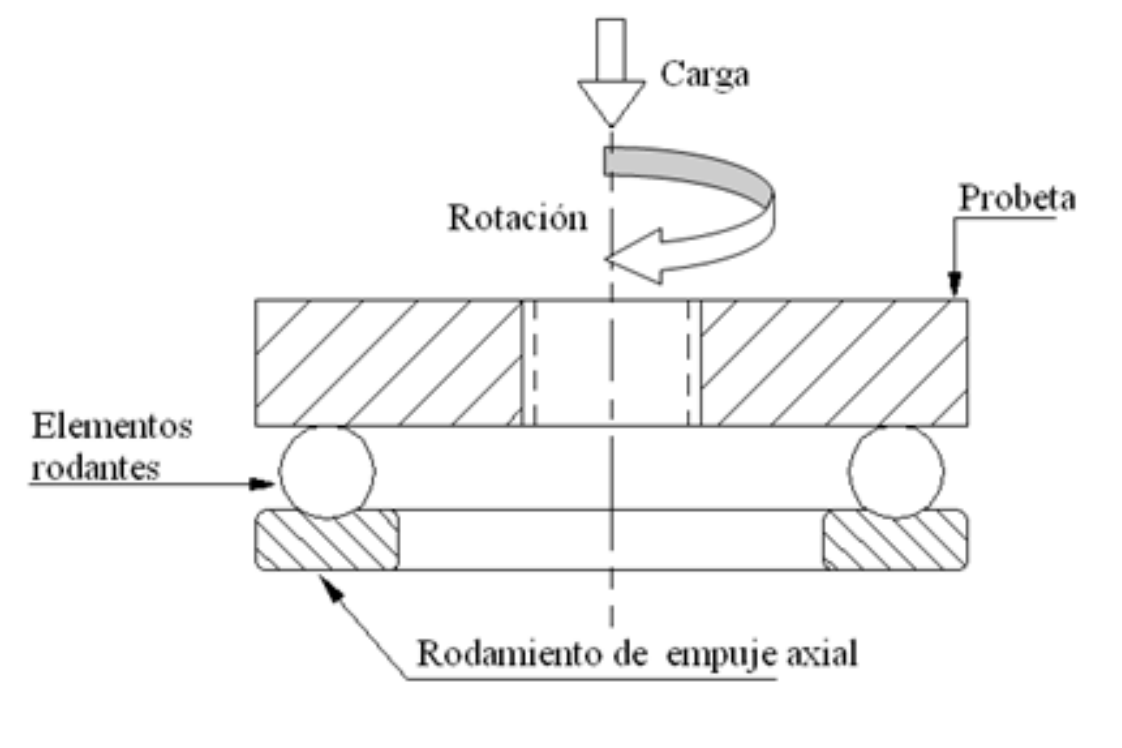

Figura 1: Configuración utilizada en los ensayos de FCR.

Los resultados de los ensayos de FCR fueron interpretados utilizando la distribución de Weibull a través la cual es posible estimar la probabilidad de falla que tiene cada variante de muestra para una cierta cantidad de ciclos de carga. La ecuación 2 corresponde a la distribución de probabilidad de falla acumulativa, $F$, en función del número de ciclos de carga, $n$. El denominado factor de forma está dado por $\beta$, e indica el tipo de distribución (normal, exponencial, etc.), mientras que $\eta$, es el valor de vida característica, en este caso, el número de ciclos correspondiente al $63,2 \%$ de probabilidad de falla.

$$
F(n)=1-e^{-\left[(n / \eta)^{\beta}\right]}
$$

Una vez finalizados los ensayos de FCR, se realizó la inspección de las probetas por microscopía óptica, microscopía electrónica de barrido (SEM) y mediante mapeos de espectrometría de dispersión de energía de rayos X (EDS). Las huellas de desgaste fueron relevadas con un rugosímetro de contacto.

Se realizó un análisis de espectro de difracción de rayos X para identificar las fases presentes, barriendo un rango angular de $2 \theta$ entre $20^{\circ}$ y $90^{\circ}$, con incrementos de $0,02^{\circ}$, empleándose radiación de $\mathrm{Cu} \mathrm{K} \alpha$ $(\lambda=1,54187 \AA)$.

\section{RESULTADOS Y DISCUSIÓN}

Los tratamientos aplicados a cada grupo de muestras permitieron evaluar el efecto del recubrimiento sobre el acero SAE 1050 ya que las propiedades del sustrato fueron las mismas para ambos grupos. El temple superficial aplicado sobre las probetas recubiertas con TiN, como era de esperar, trajo aparejada cierta degradación del film, observable a simple vista como un cambio en la tonalidad típica dorada, por otra de aspecto tornasolado más opaca y no uniforme.

El recubrimiento fue analizado mediante rayos $\mathrm{X}$, y el espectro reveló la presencia de óxido de titanio $\mathrm{TiO}_{2}$ en la superficie, explicando el cambio de color. La Fig. 2 muestra el espectro con líneas verticales para las posiciones correspondientes al rutilo $\left(\mathrm{TiO}_{2}\right)$, en coincidencia con los cinco picos de mayor intensidad ubicados en valores de $2 \theta$ de $27,4^{\circ} ; 36,1^{\circ} ; 54,23^{\circ} ; 41,1^{\circ} ; 69^{\circ}$. Es importante destacar en este punto que no se observó otro tipo de daño al recubrimiento por acción del temple superficial. No se detectó fisuración así como tampoco desprendimiento.

Al evaluar el camino de rodadura producido, por el paso repetido de los elementos rodantes en el ensayo de FCR, pueden observarse distintos tipos de daño superficial. En las etapas iniciales de los ensayos, tiene lugar la deformación plástica microscópica, plasmada en el aplastamiento de las crestas superficiales 
como se observa en la Fig. 3. Esta etapa es seguida por el desprendimiento de recubrimiento a escala microscópica, con la característica de estar alineado con los surcos superficiales provenientes de la etapa de preparación de muestras por lijado manual. La Fig. 4 a) muestra una imagen de microscopía óptica de una probeta recubierta, evidenciando desprendimientos de recubrimiento en los límites de un surco, que se identifican en la imagen como zonas brillantes. La nucleación y evolución de cráteres coincidió con lo esperado para las condiciones de trabajo impuestas, desarrollándose por el modo superficial temprano promovido por el contacto entre micro asperezas. En la Fig. 4 b) se observa la falla final, consistente en la formación de un cráter que alcanza el tamaño crítico desprendiendo material del sustrato.

En la Fig. 5 se observan imágenes SEM de probetas recubiertas obtenidas con la técnica de electrones retro difundidos (BES), mostrando la evolución del daño en el camino de rodadura (CR). Las zonas más claras provienen de la interacción del haz de electrones con elementos de mayor número atómico, en este caso el hierro $(Z=26)$ y las más oscuras con el titanio $(Z=22)$. Allí se observa, a) una red de fisuras sobre el recubrimiento en toda la huella, con desprendimiento de pequeñas porciones aisladas, b) un cráter en crecimiento dentro de una zona del CR que presenta una significativa pérdida de recubrimiento, en concordancia con las marcas de preparación manual con lija, c) el límite de la huella de desgaste donde se identifican tres tonalidades distintas, una bien clara donde el recubrimiento se desprendió completamente, otra oscura donde permanece intacto, y una tercera, de tonalidad intermedia. Para complementar esta información, se realizó un mapeo por EDS en el límite del CR, Fig. 6, observándose la presencia de titanio y ausencia de oxígeno y hierro en la mayor parte de las zonas de tono intermedio, haciendo suponer que fue removida una capa de óxido de titanio, $\mathrm{TiO}_{2}$.

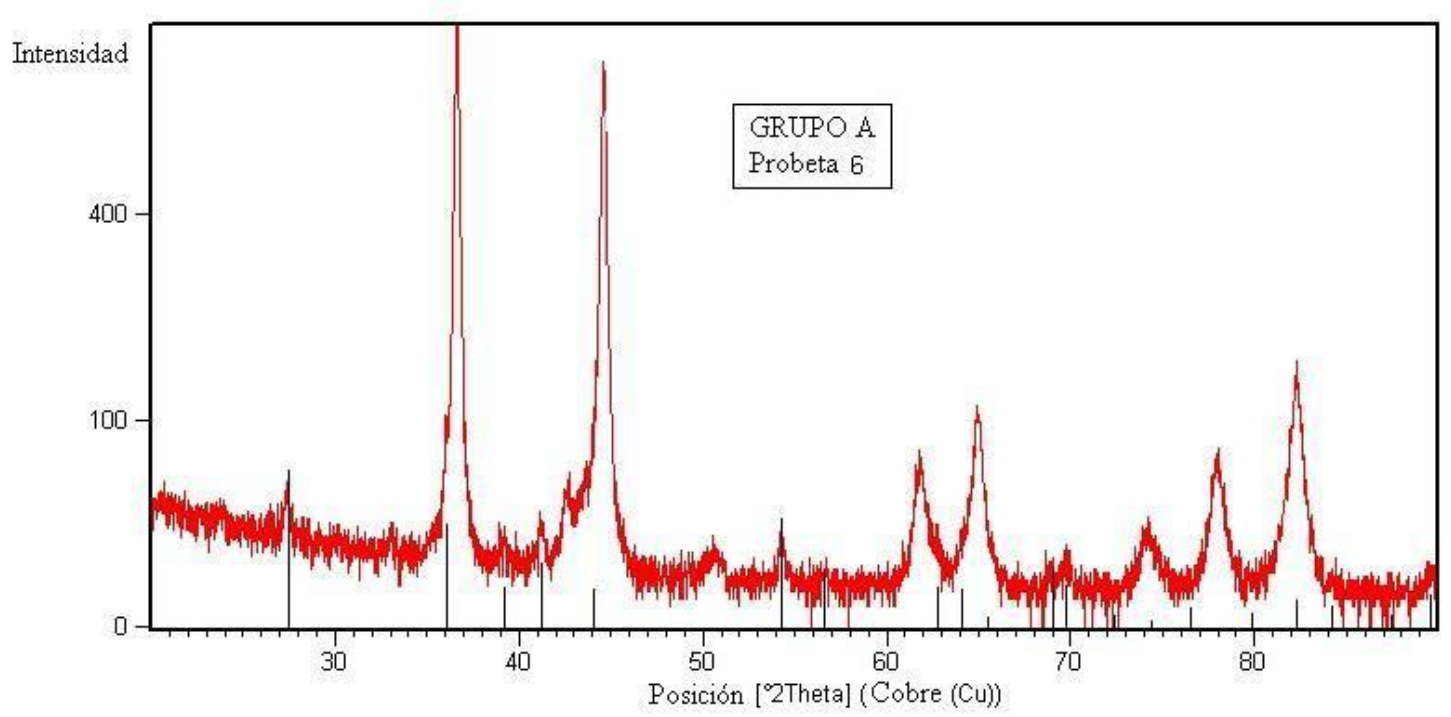

Figura 2: Espectro de DRX indicando las posiciones de los picos del rutilo $\left(\mathrm{TiO}_{2}\right)$ con líneas verticales.

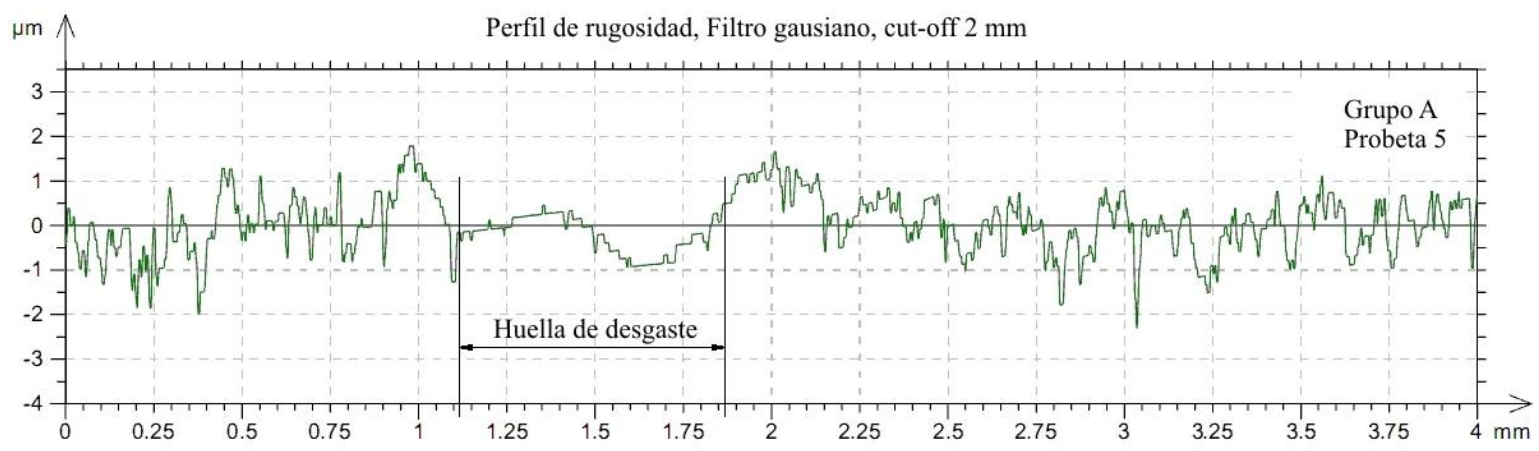

Figura 3: Perfil de rugosidad mostrando la deformación plástica de micro asperezas en la zona de rodadura. 

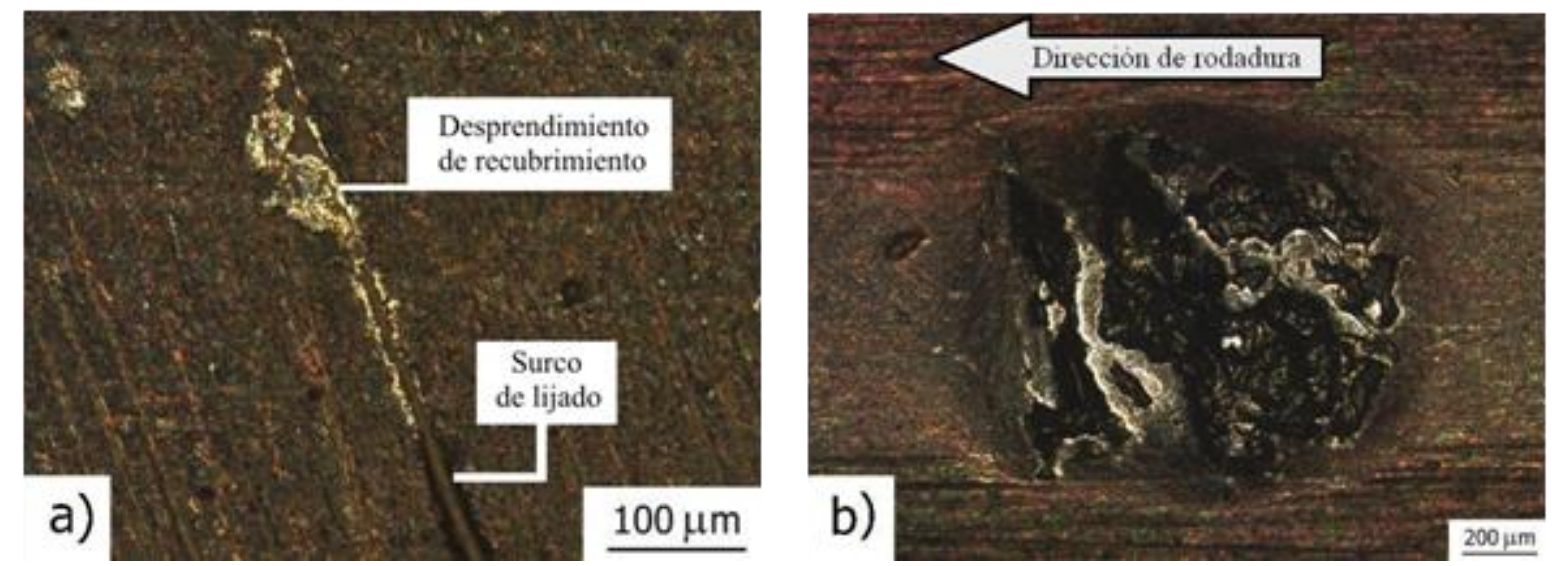

Figura 4: Daño superficial. a) Desprendimiento de recubrimiento en los límites de un surco, b) falla final después de $3,42 \times 10^{7}$ ciclos.
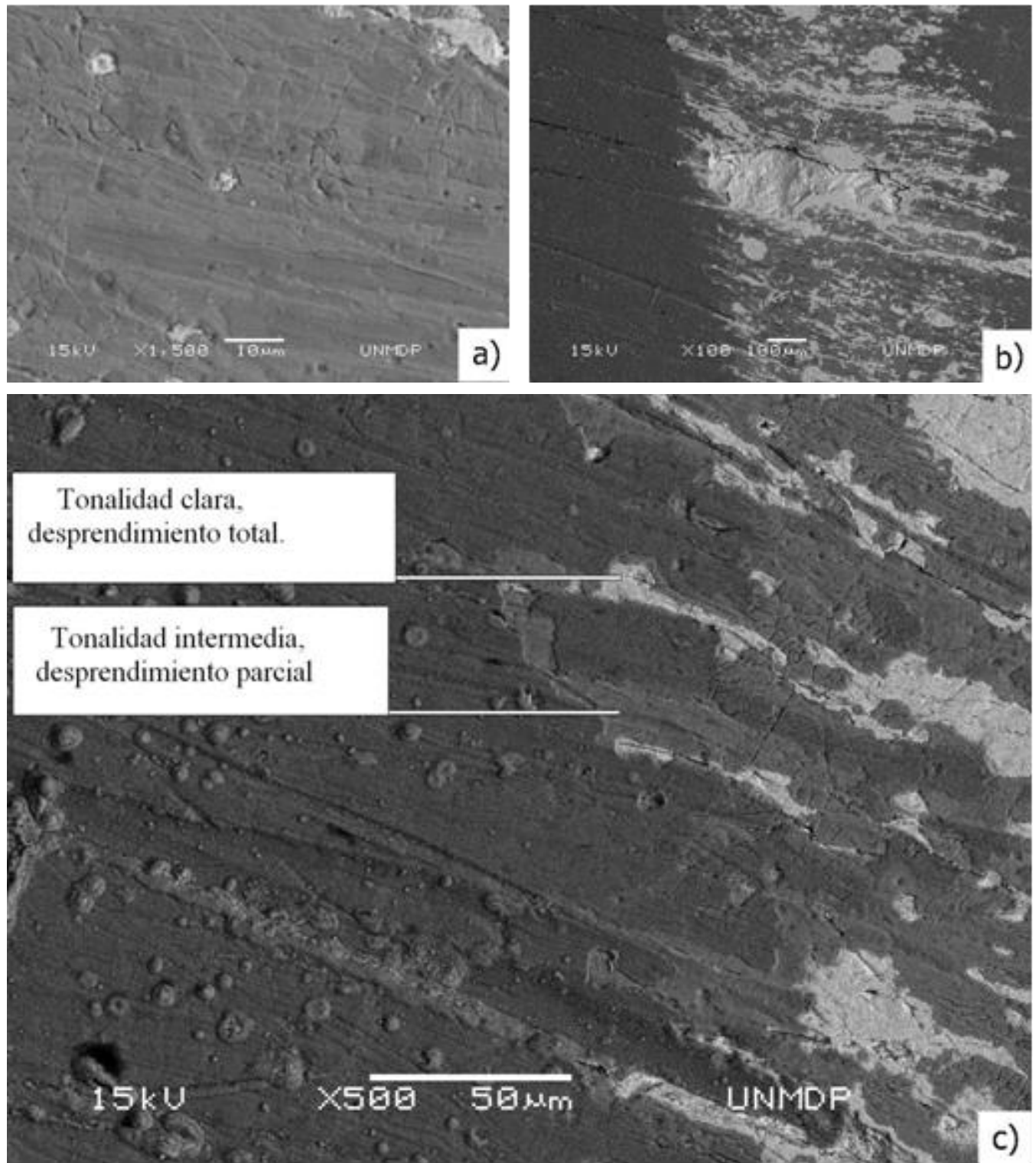

Figura 5: Imágenes SEM de electrones retro difundidos, a) red de fisuras en toda la huella, con desprendimientos aislados, b) cráter en crecimiento dentro del CR con significativa pérdida de recubrimiento, en concordancia con las marcas de preparación manual con lija, c) límite de la huella de desgaste, mostrando sectores de distintas características. 

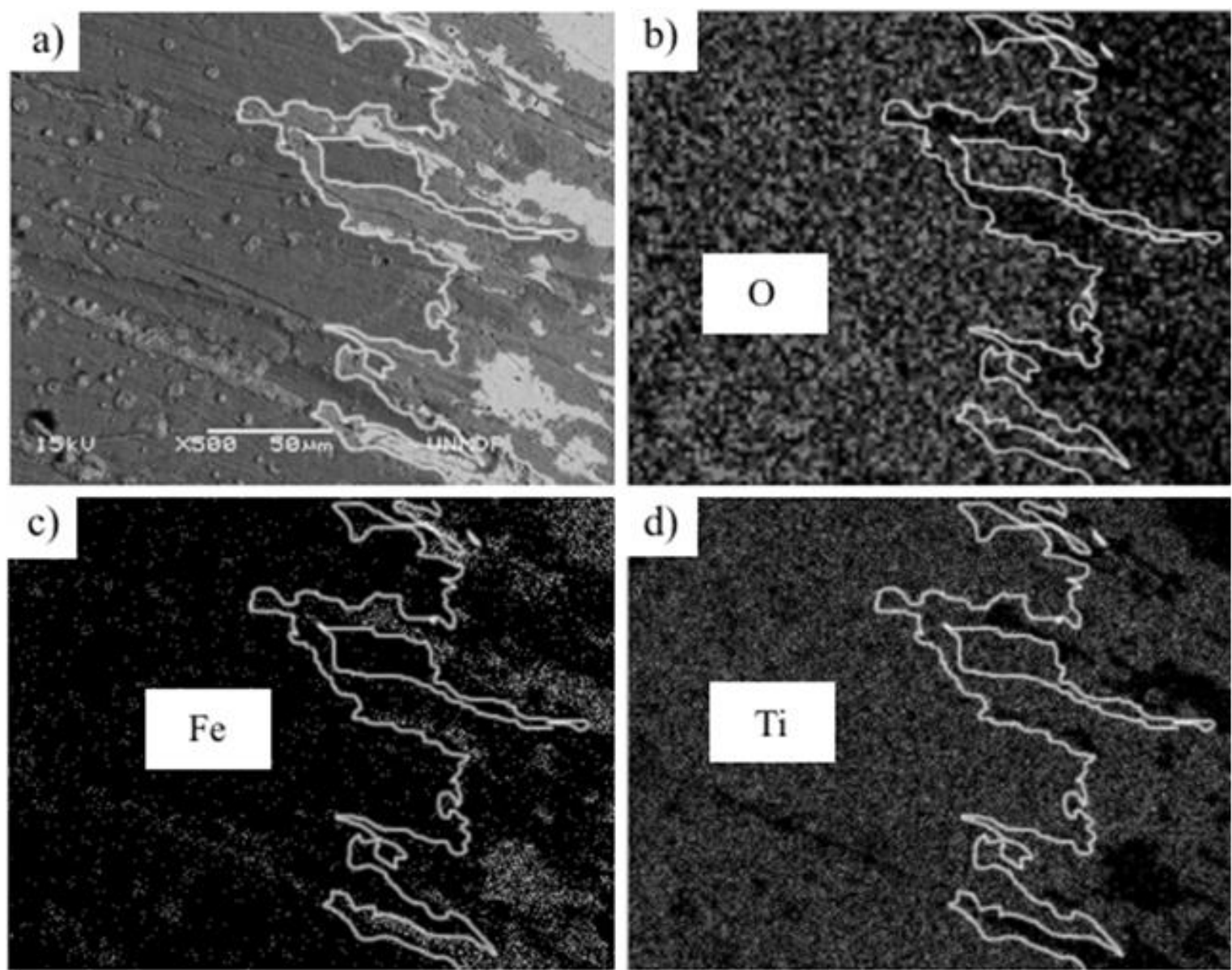

Figura 6: Se observa el límite de la zona con desprendimiento de recubrimiento. a) Imagen de EBS, b) oxígeno presente principalmente fuera de la zona con desprendimiento, c) hierro en coincidencia con las zonas de desprendimiento total, d) titanio sólo faltante donde hubo desprendimiento total.

En la Fig. 7 se muestran los resultados de los ensayos de FCR, mientras que en la tabla 1 se listan los valores de vida nominal $L_{10}$ y $L_{50}$, factor de forma, $\beta$, y vida característica, $\eta$. Si bien los valores de vida nominal resultan similares para ambos grupos, el grupo A (sin recubrimiento) muestra una distribución más estrecha y con superior valor de $\mathrm{L}_{10}$. Sin embargo la vida media que se prevé alcanzarán las probetas, aproximada por el $\mathrm{L}_{50}$, es superior para el grupo $\mathrm{B}$. Las bandas de confiabilidad mostradas, que delimitan las posibles ubicaciones de estas curvas con un 95\% de probabilidad, destacan aún más la similitud en el desempeño de ambos grupos. 


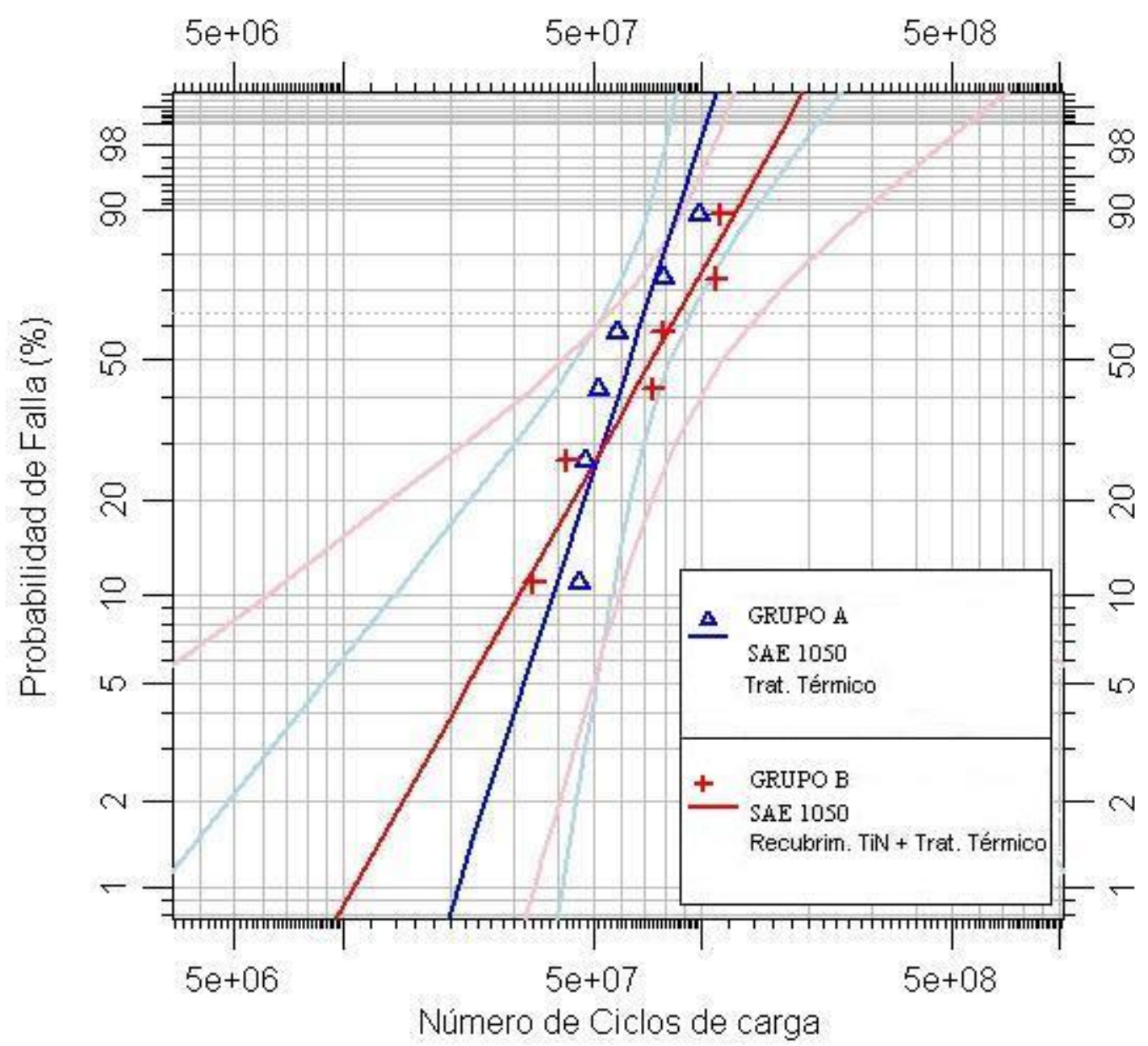

Figura 7: Resultados de los ensayos de FCR, indicando probabilidad de falla en función del número de ciclos de carga. Grupo A $(\Delta)$, Grupo B (+), bandas de confiabilidad del $95 \%$.

Tabla1: $\mathrm{L}_{10}, \mathrm{~L}_{50}, \beta$ y $\eta$ de ambos grupos.

\begin{tabular}{l|l|l|l|l}
\hline GRUPO & $\mathbf{L}_{\mathbf{1 0}}\left[\mathbf{1 0}^{\mathbf{6}}\right.$ ciclos $]$ & $\mathbf{L}_{\mathbf{5 0}}\left[\mathbf{1 0}^{\mathbf{6}}\right.$ ciclos $]$ & $\boldsymbol{\beta}$ & $\boldsymbol{\eta}$ \\
\hline A (Trat. térmico) & 38 & 64 & 3,831 & $6,96 \times 10^{7}$ \\
\hline B (Recubrim + Trat. Térmico) & 30 & 73 & 2,209 & $8,61 \times 10^{7}$ \\
\hline
\end{tabular}

\section{CONCLUSIONES}

La aplicación de recubrimientos cerámicos sobre sustratos de acero aporta diversas bondades tribológicas, sin embargo, un gradiente de dureza elevado y problemas de adhesión con el sustrato, pueden perjudicar el desempeño de estos componentes frente a la FCR. En el caso estudiado, el tratamiento realizado permite obtener un recubrimiento cerámico, sin una drástica modificación en la vida a la FCR, en sustratos de aceros de construcción.

Los resultados de los ensayos de FCR muestran que la esperanza de vida para probetas recubiertas, posteriormente templadas, es levemente superior a aquella de las probetas sin recubrir, ya que $\mathrm{L}_{50}$ resultó un $14 \%$ superior. Sin embargo, su confiabilidad resultó menor, puesto que presenta una distribución más dispersa y menor valor de $\mathrm{L}_{10}(21 \%$ menor).

El recubrimiento resultó afectado por el proceso de temple, formando dióxido de titanio (rutilo $\mathrm{TiO}_{2}$ ). El aspecto final de las piezas, una propiedad importante en algunas aplicaciones, se vio seriamente deteriorado. Parte del $\mathrm{TiO}_{2}$ fue removido durante los ensayos, implicando pérdida de material del recubrimiento y la 
generación de terceros cuerpos en el tribosistema.

Se vuelve imposible realizar mecanizados de terminación sobre las piezas ya tratadas térmicamente, ya que debería removerse el recubrimiento, esto puede resultar especialmente problemático si se tiene en cuenta que el temple puede distorsionar la geometría original una pieza.

El daño superficial observado durante los ensayos se resume en:

-Nucleación y evolución de cráteres acorde a lo esperado con los ensayos, con falla superficial temprana promovida por el contacto entre micro asperezas.

-Remoción del recubrimiento de TiN en torno a crestas de surcos provenientes del lijado manual, donde el contacto es más severo, durante las primeras etapas del ensayo.

-Desprendimiento de porciones aisladas de recubrimiento, delimitadas por una red continua de fisuras.

-Desprendimiento parcial del recubrimiento, removiéndose zonas oxidadas y permaneciendo el sustrato recubierto por TiN.

\section{AGRADECIMIENTOS}

Todos los realizadores de este trabajo agradecemos a la Universidad Nacional de Mar del Plata y a CONICET, por haber brindado su apoyo, equipos e instalaciones, al Ing Jorge Halabi y la empresa SUDOSILO S.A. por haber debatido y realizado los tratamientos superficiales a la totalidad de las probetas.

\section{BIBLIOGRAFÍA}

[1] DOWSON, D., History of Tribology, Professional Engineering Publishing, 1998.

[2] ASTM INTERNATIONAL, "Standard Terminology Relating to wear and Erosion", Designation: G40-02, West Conshohocken, PA, 19428-2959 USA, 2002.

[3] POLONSKY I. A., CHANG, T. P., KEER, L. M., et al., "A study of rolling-contact fatigue of bearing steel coated with physical vapor deposition TiN films: Coating response to cyclic contact stress and physical mechanisms underlying coating effect on the fatigue life", Wear, n. 215, pp. 191-204, 1998.

[4] CARVALHO, N. J. M., HUIS IN'T VELD, A. J., HOSSON, J. TH., "Interfacial fatigue stress in PVD TiN coated tools steels under rolling contact fatigue conditions", Surface and Coatings Technology, n. 105, pp. 109-116, 1998.

[5] STEWART, S., AHMED, R., "Rolling contact fatigue of surface coatings - a review", Wear, n. 253, pp. 1132-1144, 2002.

[6] HAMROCK B. J., DOWSON D., "Isothermal Elastohydrodynamic Lubrication of Point Contacts"; Transactions of the ASME, Journal of Lubrication Technology, pp. 264 - 276, April 1977. 